\title{
STIM1 silencing inhibits the migration and invasion of A549 cells
}

\author{
YADONG WANG ${ }^{1,2}$, HAIYU WANG ${ }^{1}$, TENG PAN $^{3}$, LI LI $^{1}$, JIANGMIN LI $^{1}$ and HAIYAN YANG ${ }^{3}$ \\ ${ }^{1}$ Department of Toxicology, Henan Center for Disease Control and Prevention, Zhengzhou, Henan 450016; \\ ${ }^{2}$ Henan Collaborative Innovation Center of Molecular Diagnosis and Laboratory Medicine, \\ Xinxiang Medical University, Xinxiang, Henan 453003; ${ }^{3}$ Department of Epidemiology, \\ School of Public Health, Zhengzhou University, Zhengzhou, Henan 450001, P.R. China
}

Received August 18, 2016; Accepted May 12, 2017

DOI: $10.3892 / \mathrm{mmr} .2017 .7010$

\begin{abstract}
The present study aimed to explore the effects of stromal interaction molecule 1 (STIM1) knockdown on the migration, invasion and metastasis of A549 cells in vitro and in vivo. Western blotting and immunohistochemistry were used to detect protein expression levels. Wound healing and Transwell invasion assays were used to assess the migratory and invasive abilities of A549 cells transfected with STIM1-specific short hairpin (sh)RNA (shSTIM1). In addition, a tail vein metastatic assay was performed. The results demonstrated that the frequency of STIM1 high-expression was significantly increased in metastatic lung cancer tissues (72.2\%) compared with in non-metastatic lung cancer tissues (33.0\%). STIM1 knockdown inhibited A549 cell migration and invasion in vitro and tumor metastasis in vivo. The protein expression levels of Snail1, Vimentin, matrix metalloproteinase (MMP) 2 and MMP9 were markedly decreased in A549-shSTIM1 compared with in A549 cells transfected with control shRNA (shcon). In addition, the protein expression levels of E-cadherin were markedly increased in A549-shSTIM1 cells compared with in A549-shcon cells. These results suggested that STIM1 knockdown may inhibit the migration and invasion of A549 cells in vitro, and metastasis in vivo.
\end{abstract}

\section{Introduction}

An estimated 1.8 million new cases of lung cancer were diagnosed in 2012, which accounted for $\sim 13 \%$ of total cancer

Correspondence to: Dr Yadong Wang, Department of Toxicology, Henan Center for Disease Control and Prevention, 105 South Nongye Road, Zhengzhou, Henan 450016, P.R. China

E-mail: wangyd76@163.com

Dr Haiyan Yang, Department of Epidemiology, School of Public Health, Zhengzhou University, 100 Science Road, Zhengzhou, Henan 450001, P.R. China

E-mail: yhy@zzu.edu.cn

Key words: stromal interaction molecule 1, lung cancer, migration, invasion, A549 cells diagnoses. In addition, lung cancer was the most frequently diagnosed cancer and the leading cause of cancer-associated mortality among men in 2012. Among females, lung cancer was the leading cause of cancer-associated mortality in more developed countries, and the second leading cause of cancer-associated mortality in less developed countries (1). Despite improvements being made in the diagnosis and treatment of lung cancer in recent decades, the 5-year survival rate of patients with lung cancer is still relatively low (2-4). Tumor metastasis is one of the major causes of lung cancer-associated motility. Therefore, in order to improve the treatment of lung cancer, the mechanism underlying lung cancer metastasis should be fully understood to facilitate the establishment of methods that suppress tumor metastasis.

Stromal interaction molecule 1 (STIM1) was initially identified as a novel human gene, which is mapped to a region of chromosome $11 \mathrm{p} 15.5$ (5). The STIM1 protein mediates $\mathrm{Ca}^{2+}$ influx, following depletion of intracellular $\mathrm{Ca}^{2+}$ stores, via gating of store-operated $\mathrm{Ca}^{2+}$ influx channels. STIM1 is critical for the development and functioning of numerous cell types, including lymphocytes, skeletal and smooth muscle myoblasts, adipocytes and neurons, and can interact with various signaling proteins and pathways in a cell- and tissue-type specific manner (6). STIM1 was initially identified as an antimetastatic gene in melanoma cells by Suyama et al (7); however, recent studies have reported that STIM1 exhibits a metastatic function. Yang et al (8) observed that STIM1 silencing inhibited the migration and metastasis of breast cancer cells. Chen et al (9) revealed that a significantly poorer clinical outcome in primary tumors was associated with STIM1 upregulation; STIM1 overexpression markedly enhanced local spread and angiogenesis, and promoted cancer cell migration and invasion. Additional studies have indicated that knocking down STIM1 may result in reduced cancer metastasis in hepatocellular carcinoma $(10)$, melanoma $(11,12)$ and colorectal cancer (13).

The potential role of STIM1 in lung cancer metastasis remains to be elucidated. To the best of our knowledge, one previously published paper reported that STIM1 expression was increased in non-small cell lung cancer (NSCLC) tissues compared with in adjacent non-neoplastic lung tissues (14). To further understand the potential role of STIM1 in lung cancer metastasis, the present study analyzed the expression of STIM1 in metastatic lung cancer tissues and non-metastatic 
lung cancer tissues. In addition, the effects of STIM1 silencing on A549 cell migration and invasion in vitro, and on metastasis in vivo, were investigated.

\section{Materials and methods}

Cell culture. STIM1 short hairpin (sh)RNA (h) lentiviral particles (sc-76589-V) and control shRNA lentiviral particles-A (sc-108080) were purchased from Santa Cruz Biotechnology, Inc. (Dallas, TX, USA). A549 cells (15) were obtained from American Type Culture Collection (Manassas, VA, USA) and infected with lentiviral particles according to the manufacturer's protocol (Santa Cruz Biotechnology, Inc.). The infected A549 cells were selected with $10 \mu \mathrm{g} / \mathrm{ml}$ puromycin (Gibco; Thermo Fisher Scientific, Inc., Waltham, MA, USA). The stable clone infected with STIM1 shRNA (h) lentiviral particles was termed A549-shSTIM1 and the stable clone infected with control shRNA lentiviral particles was termed A549-shcon. All cell lines were cultured in RPMI-1640 medium (Beijing Solarbio Science \& Technology Co., Ltd., Beijing, China) supplemented with penicillin $(100 \mathrm{U} / \mathrm{ml})$, streptomycin $(100 \mu \mathrm{g} / \mathrm{ml}), 10 \%$ fetal bovine serum (FBS) (Hyclone; GE Healthcare Life Sciences, Logan, UT, USA) and $2 \mathrm{mM} \mathrm{L}$-glutamine at $37^{\circ} \mathrm{C}$ in an atmosphere containing $5 \% \mathrm{CO}_{2}$.

Biological samples. A total of 49 lung cancer tissue samples were obtained from patients between January and December 2014, following surgical resection at the First Hospital Affiliated to Zhengzhou University (Zhengzhou, China). None of the patients received radiation therapy or chemotherapy prior to surgery. Histological type and cell differentiation grade were determined according to the World Health Organization criteria (16). Written informed consent for participation in the present study was obtained from all patients prior to surgery. The present study was approved by the Ethics Committee of the First Hospital Affiliated to Zhengzhou University, and the associated methods were conducted in accordance with the approved guidelines.

Wound healing assay. A wound healing assay was performed according to the method described in our previous study (17). Briefly, monolayer cells were wounded by scratching the surface of each well in a 6-well plate as uniformly as possible with a $200 \mu 1$ pipette tip. The wells were then rinsed with phosphate-buffered saline (PBS) three times and were incubated at $37^{\circ} \mathrm{C}$ for $48 \mathrm{~h}$. Images of the initial wound, and the movement of cells into the scratched area, were captured using a Leica DM IL LED inverted microscope equipped with a digital imaging system (Leica Microsystems GmbH, Wetzlar, Germany) at 0 and $48 \mathrm{~h}$. The wound width of 5 random views was measured. The healing width was calculated as wound width at $0 \mathrm{~h}$ minus wound width at $48 \mathrm{~h}$, and was normalized to A549-shcon cells (17).

Transwell invasion assay. The cell invasion assay was performed using a 24-well Transwell chamber (Corning Incorporation, Corning, NY, USA). A549-shSTIM1 and A549-shcon cells were seeded at a density of $4 \times 10^{4}$ cells into the upper chamber (pore size, $8 \mu \mathrm{m}$ ), which was precoated with
Matrigel (BD Biosciences, Franklin Lakes, NJ, USA). The lower chamber was filled with $600 \mu \mathrm{l}$ RPMI-1640 containing $10 \%$ FBS. Following a $24 \mathrm{~h}$ incubation at $37^{\circ} \mathrm{C}$, cells on the upper-side of the membrane were removed using clean swabs, and cells on the underside were viewed and counted under a Leica DM IL LED inverted microscope (Leica Microsystems $\mathrm{GmbH})$. The number of invaded cells was counted in 5 randomly selected fields (17).

Tail vein metastatic assay. All experimental procedures involving animals were performed in accordance with the Guide for the Care and Use of Laboratory Animals $(18,19)$, and the study was approved by the Ethics Committee of Henan Center for Disease Control and Prevention (Zhengzhou, China). Female BALB/c nude mice (age, 4 weeks; weight, $20 \pm 2 \mathrm{~g}$ ) were purchased from Vital River Laboratory Animal Technology Co.,Ltd. (Beijing, China), were housed in a specific pathogen-free environment at a controlled temperature of $22 \pm 2^{\circ} \mathrm{C}$ and $50-60 \%$ relative humidity, under 12 -h light/dark cycles and were provided with free access to food and water. A549-shSTIM1 cells and A549-shcon cells were harvested, washed twice with PBS, and suspended in PBS. A total of 5 mice per group received $5 \times 10^{6}$ cells in $150 \mu \mathrm{l}$ PBS by tail vein injection. The mice were sacrificed at 12 weeks post-injection. Subsequently, the lung tissues were histologically examined for metastases under a Zeiss Axioskop 40 microscope (Zeiss AG, Oberkochen, Germany) (13).

Western blot analysis. Total protein was extracted from cultured cells with lysis buffer solution supplemented with protease and phosphatase inhibitors (Pierce; Thermo Fisher Scientific, Inc.), and protein concentration was quantified using a bicinchoninic acid protein assay (Pierce; Thermo Fisher Scientific, Inc.). Each protein sample $(30 \mu \mathrm{g})$ was separated by $10 \%$ SDS-PAGE and was then transferred to nitrocellulose membranes (Pall Life Sciences, Port Washington, NY, USA). After blocking with $5 \%$ bovine serum albumin (Beyotime Institute of Biotechnology, Haimen, China) in Tris-buffered saline containing $0.1 \%$ Tween-20 at room temperature for $1 \mathrm{~h}$, the membrane was incubated with primary antibodies at a 1:1,000 dilution overnight at $4^{\circ} \mathrm{C}$. STIM1 (sc-68897), matrix metalloproteinase (MMP)2 (sc-10736), MMP9 (sc-10737), $\mathrm{N}$-cadherin (sc-7939) and E-cadherin (sc-7870) antibodies were purchased from Santa Cruz Biotechnology, Inc. Snaill (\#3879S) and Vimentin (\#5741S) antibodies were purchased from Cell Signaling Technology, Inc. (Danvers, MA, USA). After incubation with horseradish peroxidase (HRP)-coupled anti-rabbit-immunglobulin G (ZB-2301; 1:5,000; ZSGB-BIO, Beijing, China) and HRP-coupled anti-mouse-immunglobulin G (ZB-2305; 1:5,000; ZSGB-BIO) at room temperature for $1 \mathrm{~h}$, the protein bands were visualized using Bio-Rad Clarity ${ }^{\mathrm{TM}}$ western enhanced chemiluminescence substrate (Bio-Rad Laboratories, Inc., Hercules, CA, USA) and the ChemiDoc ${ }^{\mathrm{TM}}$ $\mathrm{XRS}^{+}$Imaging system (Bio-Rad Laboratories, Inc.). Anti- $\beta$-actin (1:1,000; sc-47778; Santa Cruz Biotechnology, Inc.) was used as a loading control.

Immunohistochemistry. Immunohistochemistry of human lung cancer tissue samples was performed using a streptavidin rabbit \& mouse HRP kit (\#CW2069; Beijing ComWin 
Biotech Co., Ltd., Beijing, China) according to standard procedures. Briefly, following deparaffinization, antigen retrieval was performed with sodium citrate buffer and boiling for $15 \mathrm{~min}$. Slides were blocked with endogenous peroxidase blocking buffer and normal goat serum (supplied in the kit) at room temperature for $10 \mathrm{~min}$ each. Slides were washed with PBS and incubated with anti-STIM1 primary antibody (sc-68897; 1:100; Santa Cruz Biotechnology, Inc.) at $4^{\circ} \mathrm{C}$ overnight, and then incubated with biotinylated secondary antibody and HRP-labeled streptavidin (supplied in the kit) at room temperature for $10 \mathrm{~min}$ each. Staining was performed with $100 \mu \mathrm{l}$ diaminobenzidine at room temperature for $1 \mathrm{~min}$. The intensity of STIM1 staining was scored as 0 (no signal), 1 (weak), 2 (moderate) and 3 (marked). Scores of the percentage of positive cells were assigned as $0(<5 \%)$, $1(5-25 \%), 2(26-50 \%)$ and $3(>51 \%)$. The scores of each view were multiplied to give a final score of $0-9$, and the final score of one sample was the mean of 5 microscopic fields. Tumors were finally determined as low-expression (score: $0-1$ ) and high-expression (score: 2-9) (20).

Statistical analysis. Data are presented as the mean \pm standard deviation of 3 independent experiments. Statistical significance was estimated using SPSS version 13.0 software (SPSS, Inc., Chicago, IL, USA). Data were analyzed by Student's t-test and $\chi^{2}$ test; all tests were two-sided. $\mathrm{P}<0.05$ was considered to indicate a statistically significant difference.

\section{Results}

STIM1 expression in human lung cancer tissues. The clinicopathological characteristics of the patients with lung cancer are presented in Table I. To determine the difference in STIM1 protein expression between metastatic lung cancer tissues and non-metastatic lung cancer tissues, immunohistochemistry was conducted. The results demonstrated that the frequency of STIM1 high-expression was $72.2 \%(13 / 18)$ in metastatic lung cancer tissues, which was significantly higher than that in non-metastatic lung cancer tissues ( $\mathrm{P}=0.013$; Table II). Representative images (Fig. 1) exhibit the high-expression (Fig. 1A) and low-expression (Fig. 1B) of STIM1 in lung cancer tissues.

Effects of STIM1 silencing on A549 cell migration and invasion in vitro. To evaluate the effects of STIM1 silencing on the migration of A549 cells, a wound healing assay was used to determine the migratory ability of A549 cells. Knockdown of STIM1 expression was confirmed by western blot analysis (Fig. 2A). The results of the wound healing assay indicated that the cells migrated more slowly to close the scratched wounds in the A549-shSTIM1 group compared with the A549-shcon group (Fig. 2B). Furthermore, a Transwell invasion assay was conducted to evaluate the effects of STIM1 silencing on the invasive abilities of A549 cells. The results demonstrated that the ability of A549 cells to invade through the Matrigel matrix was significantly decreased in the A549-shSTIM1 group compared with the A549-shcon group (Fig. 2C). Taken together, these results suggested that STIM1 silencing may inhibit the migratory and invasive abilities of A549 cells in vitro.
Table I. Clinicopathological characteristics of patients with lung cancer.

\begin{tabular}{lc}
\hline Characteristic & Number \\
\hline Male & 39 \\
$\geq 60$ years & 26 \\
$<60$ years & 13 \\
Female & 10 \\
$\geq 60$ years & 6 \\
$<60$ years & 4 \\
Histological type & \\
Adenocarcinoma & 20 \\
Squamous cell carcinoma & 26 \\
Small cell carcinoma & 3 \\
Grade & \\
G1 & 10 \\
G2 & 29 \\
G3 & 10 \\
Metastasis & \\
Yes & 18 \\
No & 31 \\
\hline
\end{tabular}

Table II. Frequency of stromal interaction molecule 1 high-expression and low-expression in metastatic and non-metastatic lung cancer tissues.

\begin{tabular}{lcc}
\hline Expression & $\begin{array}{c}\text { Metastatic } \\
\text { lung cancer }\end{array}$ & $\begin{array}{c}\text { Non-metastatic } \\
\text { lung cancer }\end{array}$ \\
\hline High-expression & 13 & 11 \\
Low-expression & 5 & 20 \\
\hline
\end{tabular}

$\chi^{2}=6.151, P=0.013$.

Effects of STIM1 silencing on A549 metastasis in vivo. Since STIM1 silencing was revealed to inhibit A549 cell migration and invasion in vitro, the present study then injected A549-shSTIM1 and A549-shcon cells into nude mice through the lateral tail vein. The results of a metastatic assay indicated that the number of metastatic lung nodules was significantly decreased in the A549-shSTIM1 group compared with the A549-shcon group (Fig. 3).

STIM1 silencing downregulates the protein expression levels of Snail1, MMP2, MMP9 and Vimentin, and upregulates the protein expression levels of E-cadherin. Since specific proteins, such as Snail1, MMP2, MMP9, $\mathrm{N}$-cadherin, Vimentin and E-cadherin, are associated with the progression and metastasis of lung cancer (21-25), the present study aimed to determine whether STIM1 silencing could affect the expression levels of these proteins. As shown in Fig. 4, western blot analysis indicated that the protein expression levels of Snail1, MMP2, MMP9 and Vimentin were markedly decreased in A549-shSTIM1 cells 

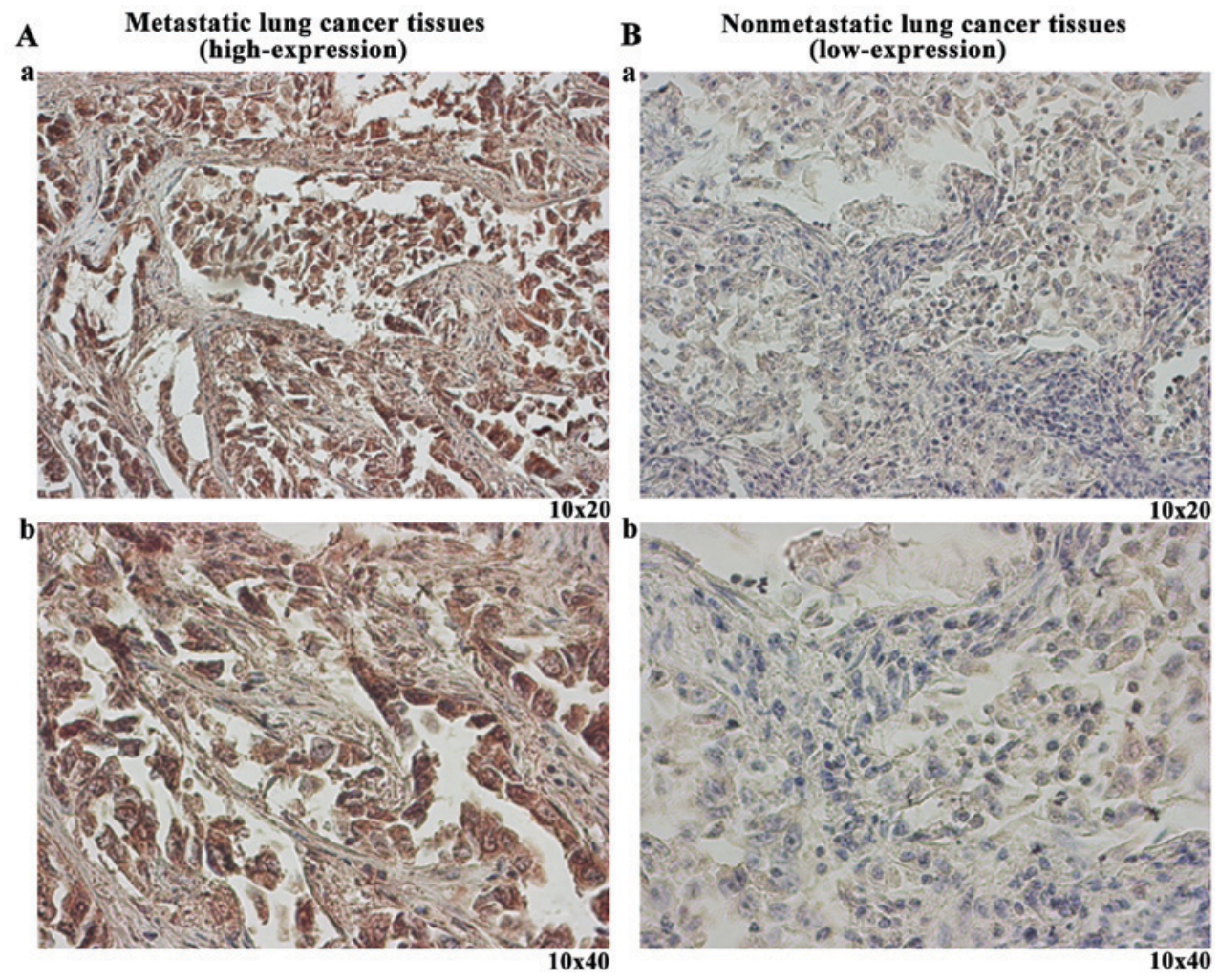

Figure 1. Representative images of stromal interaction molecule 1 (A) high-expression and (B) low-expression in lung cancer tissues. (A-a and B-a) Magnification, 10x20; (A-b and B-b) magnification, 10x40.

A

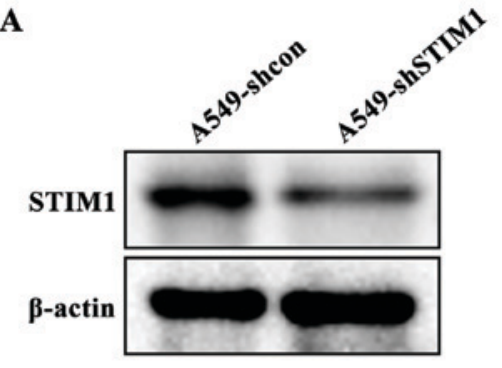

C

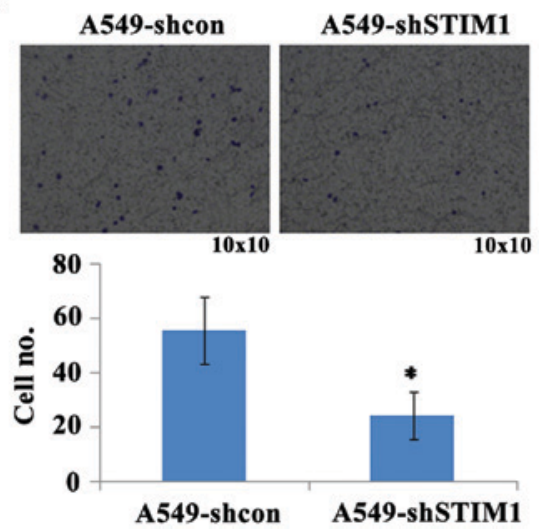

B

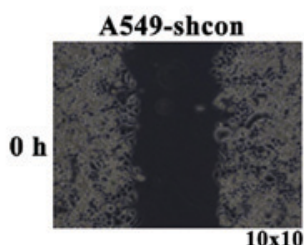

A549-shSTIM1
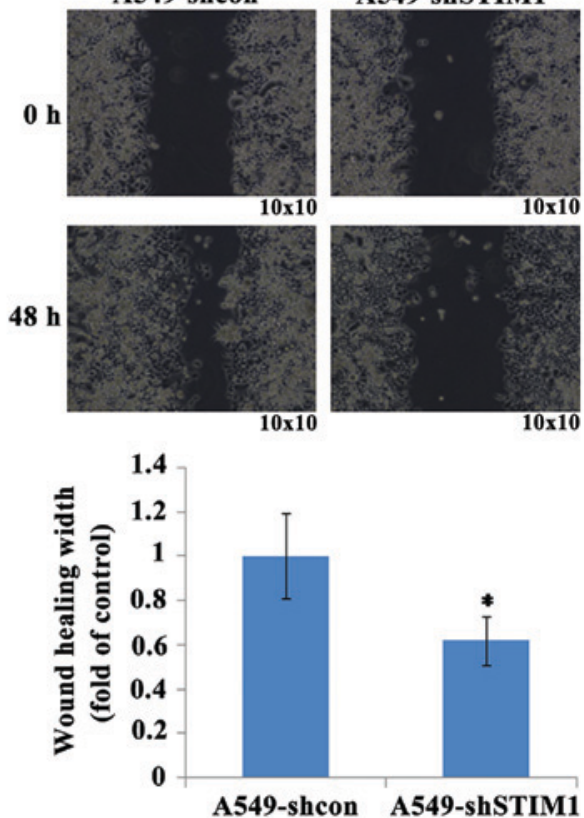

Figure 2. Effects of STIM1 silencing on the migration and invasion of A549 cells. (A) Knockdown of STIM1 expression was confirmed by western blot analysis. (B) Cell migration to close the scratched wounds was reduced in the A549-shSTIM1 cells compared with the A549-shcon cells. (C) Reduced invasion through the Matrigel matrix was observed in the A549-shSTIM1 cells compared with the A549-shcon cells. " $\mathrm{P}<0.05$. shcon, control short hairpin RNA; shSTIM1, STIM1 short hairpin RNA; STIM1, stromal interaction molecule 1.

compared with in A549-shcon cells. However, the protein expression levels of E-cadherin were markedly increased in A549-shSTIM1 cells compared with in A549-shcon cells.
There were no obvious alterations in the protein expression levels of N-cadherin between A549-shSTIM1 cells and A549-shcon cells. 

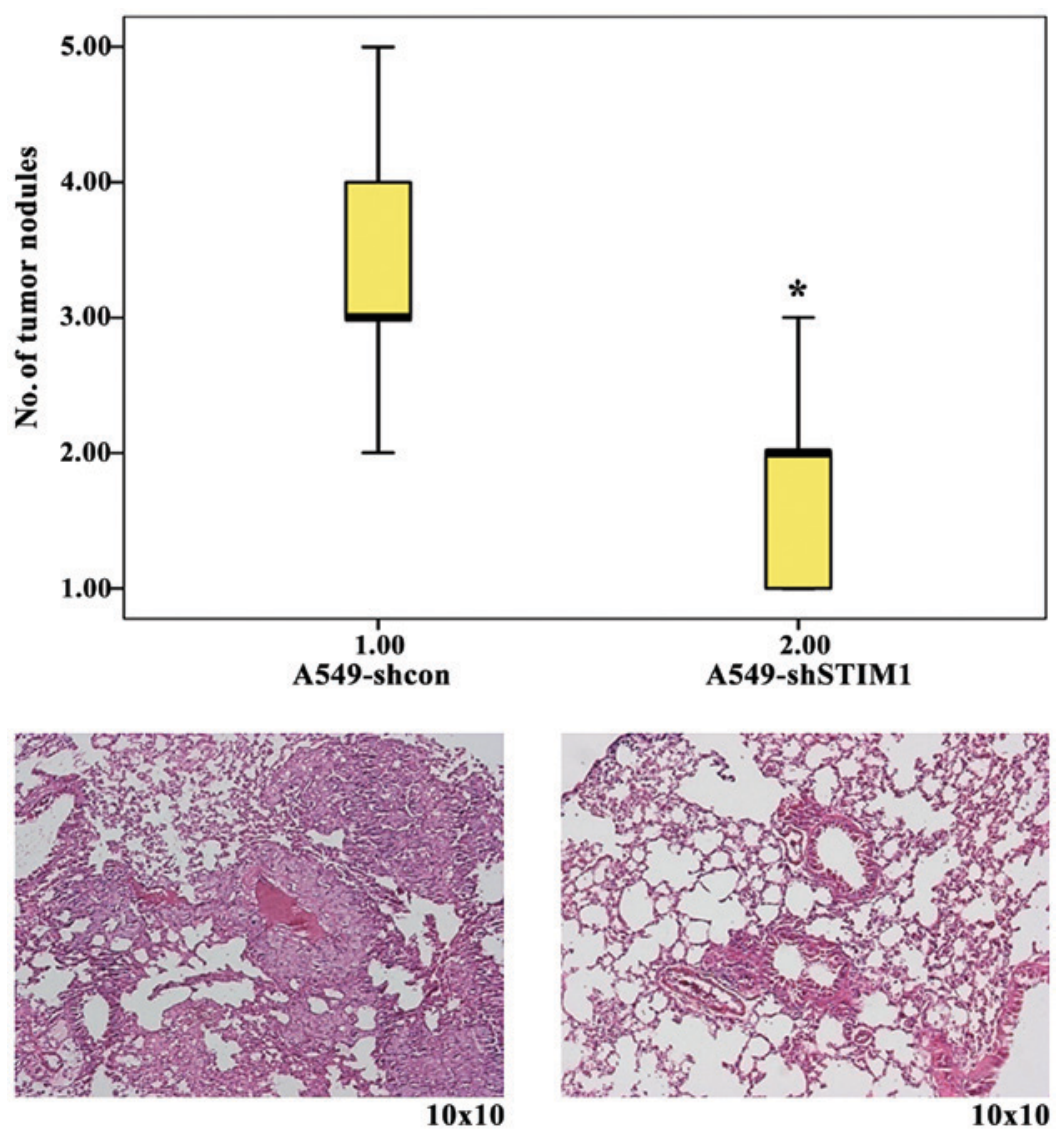

Figure 3.Effects of STIM1 silencing on A549 cell metastasis in vivo. The number of metastatic lung nodules was significantly reduced in the A549-shSTIM1 group compared with the control group. "P<0.05. shcon, control short hairpin RNA; shSTIM1, STIM1 short hairpin RNA; STIM1, stromal interaction molecule 1.

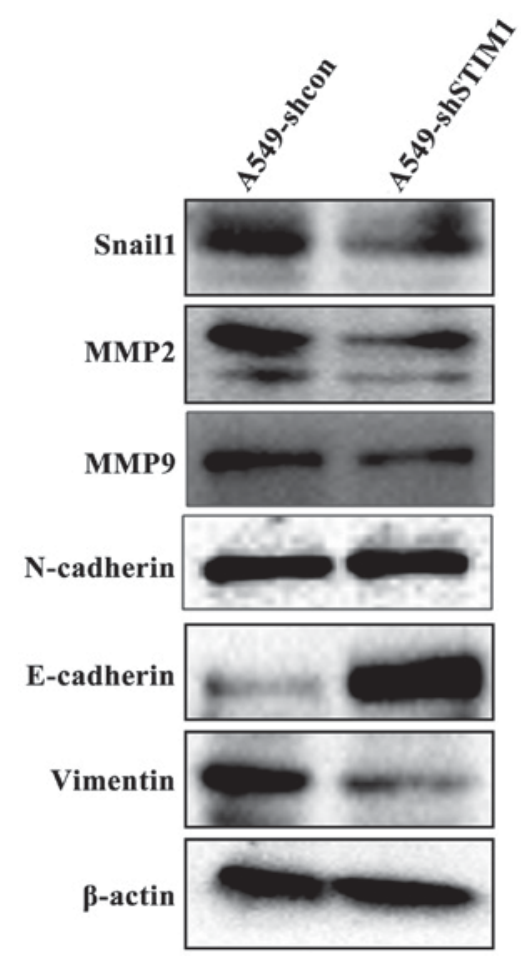

Figure 4. Effects of STIM1 silencing on the expression of several proteins. Western blot analysis indicated that STIM1 silencing decreased the protein expression levels of Snail1, MMP2, MMP9 and Vimentin, and increased the protein expression levels of E-cadherin. MMP, matrix metalloproteinase; shcon, control short hairpin RNA; shSTIM1, STIM1 short hairpin RNA; STIM1, stromal interaction molecule 1.

\section{Discussion}

STIM1 was initially identified as an antimetastatic gene, since STIM1 silencing resulted in the accelerated mobility of melanoma cells in an in vitro scratch-wound assay (7); however, subsequent studies reported conflicting results. Umemura et al (11) reported that STIM1 knockdown inhibited cell migration in metastatic cell lines and decreased the number of metastatic colonies in mouse lung tissues. Yang et al (8) demonstrated that silencing STIM1 inhibited the migration and metastasis of breast cancer cells by suppressing focal adhesion turnover. In addition, Chen et al (9) reported that the expression levels of STIM1 were significantly associated with the risk of cervical cancer metastasis and survival. Silencing STIM1 attenuated the endogenous migration of cervical cancer cells, whereas STIM1 overexpression enhanced cervical cancer cell migration and invasion. Zhang et al (13) reported that the enhanced expression of STIM1 promoted colorectal cancer cell metastasis in vitro and in vivo, whereas silencing STIM1 with small interfering RNA reduced metastasis. Furthermore, ectopic expression of STIM1 in colorectal cancer cells induced epithelial to mesenchymal transition (EMT), whereas silencing STIM1 exerted discordant effects (13). Taken together, the potential role of STIM1 in tumor metastasis is inconsistent among different tumor types; the detailed reasons why have yet to be elucidated. One of the main causes of lung cancer-associated mortality is tumor metastasis; however, it has yet to be determined whether STIM1 serves an important role in lung 
cancer metastasis. Therefore, the present study aimed to determine the role of STIM1 in lung cancer metastasis.

The present study demonstrated that the frequency of STIM1 high-expression was increased in metastatic lung cancer tissues compared with in non-metastatic lung cancer tissues, thus suggesting that STIM1 overexpression may promote lung cancer metastasis. To confirm the findings demonstrated in human samples, the effects of STIM1 silencing on the invasive and migratory abilities of A549 cells were investigated in vitro. The results indicated that STIM1 silencing inhibited the migratory and invasive capabilities of A549 cells, as determined using wound healing and Transwell invasion assays. These findings supported the aforementioned observations to a certain extent. In an animal model, the present study demonstrated that STIM1 silencing inhibited the metastasis of A549 cells in vivo, which further verified the role of STIM1 in lung cancer metastasis.

The present study indicated that STIM1 was expressed not only in cancer cells but also in stromal cells. Previous studies (26-28) have reported that the tumor stroma serves a crucial role in tumorigenesis. The tumor stroma contains increased amounts of inflammatory infiltrates, an increased microvessel density with dysfunctional lymphatics and blood vessels, and a denser extracellular matrix with reactive fibroblasts (29). Throughout the entire process of cancer etiology, progression and metastasis, the microenvironment of the local host tissue may be considered an active participant. Invasion occurs within the tumor-host microecology, where stroma and tumor cells exchange enzymes and cytokines that modify the local extracellular matrix, stimulate migration, and promote proliferation and survival (30). However, it remains unclear how STIM1 expression is regulated in cancer tissues, which should be explored in future studies.

E-cadherin is a calcium-dependent, epithelial cell adhesion molecule, the reduced expression of which has been associated with increased lymph node metastasis in NSCLC (31-33). Transfection of E-cadherin cDNA in human lung tumor cells has previously been reported to reduce the invasive potential of tumors (34), and overexpression of E-cadherin in NSCLC cell lines may inhibit cell migration (35). Vimentin is an intermediate filament protein whose expression is correlated with increased metastatic disease, reduced patient survival and poor prognosis in lung cancer $(36,37)$. Vimentin expression is also associated with prognosis via alteration of the invasive ability of NSCLC cells (38). Vimentin depletion inhibited lung cancer cell migration and invasion in vitro, and metastasis in vivo (39). In the present study, STIM1 silencing downregulated Vimentin protein expression and upregulated E-cadherin protein expression. A previous study demonstrated that STIM1 silencing downregulated Vimentin expression and upregulated E-cadherin expression in colorectal cancer cells; however, overexpression of STIM1 had the opposite effect (13). Taken together, these findings supported the hypothesis that STIM1 may regulate the expression of Vimentin and E-cadherin in various cell lines. However, there is no significant difference in cell morphology between A549-shcon cells and A549-shSTIM1 cells (data not shown). The discrepancy in the alterations of EMT markers and cell phenotype observed in the present study should be explored in future studies.

MMPs are a class of proteolytic enzymes that are closely associated with tumor invasion and metastasis. MMP2 and
MMP9 are the major enzymes that degrade type IV collagen, which serve important roles in lung cancer metastasis (40-42). A previous study demonstrated that increased MMP2 expression may serve as an independent prognosis factor in NSCLC, which is closely associated with clinical stage, pathological grade, lymphatic metastasis and prognosis (43). MicroRNA-129 may regulate MMP9 to control metastasis of NSCLC (44). In the present study, STIM1 silencing decreased the protein expression levels of MMP2 and MMP9 in A549 cells. A previous study reported that STIM1 knockdown could reduce the levels of secreted MMP2 in melanoma cells in vitro (12). Snail1 is a zinc-finger transcription factor that contains a highly conserved C-terminal region comprising 4-6 zinc fingers, which serves as the DNA-binding domain that recognize consensus E-box type elements $(45,46)$. Snaill has been reported to serve a key role in lung cancer metastasis and progression (46-48). Furthermore, previous studies have indicated that Snaill may regulate the expression of E-cadherin, Vimentin, MMP2 and MMP9 in lung cancer cell lines $(48,49)$. The present study observed that STIM1 silencing downregulated the expression of Snaill protein; however, the molecular mechanism underlying STIM1-mediated regulation of Snaill expression remains unclear, and should be further investigated in future studies.

In conclusion, the present study suggested that STIM1 silencing inhibited the migration and invasion of A549 cells in vitro, and lung cancer cell metastasis in vivo. The present study extends the knowledge regarding lung cancer progression and suggests that STIM1 may be a potential therapeutic target for the treatment of human lung cancer.

\section{Acknowledgements}

The present study was supported by grants from the National NaturalScienceFoundation ofChina(grantno.U1404815)and the Henan Collaborative Innovation Center of Molecular Diagnosis and Laboratory Medicine (grant no. XTCX-2015-PY7).

\section{References}

1. Torre LA, Bray F, Siegel RL, Ferlay J, Lortet-Tieulent J and Jemal A: Global cancer statistics, 2012. CA Cancer J Clin 65: 87-108, 2015.

2. Cui T, Srivastava AK, Han C, Yang L, Zhao R, Zou N, Qu M, Duan W, Zhang X and Wang QE: XPC inhibits NSCLC cell proliferation and migration by enhancing E-Cadherin expression. Oncotarget 6: 10060-10072, 2015.

3. Osarogiagbon RU, Lin CC, Smeltzer MP and Jemal A: Prevalence, prognostic implications, and survival modulators of incompletely resected non-small cell lung cancer in the U.S. national cancer data base. J Thorac Oncol 11: e5-e16, 2016.

4. Lin JJ, Cardarella S, Lydon CA, Dahlberg SE, Jackman DM, Jänne PA and Johnson BE: Five-year survival in EGFR-mutant metastatic lung adenocarcinoma treated with EGFR-TKIs. J Thorac Oncol 11: 556-565, 2016.

5. Parker NJ, Begley CG, Smith PJ and Fox RM: Molecular cloning of a novel human gene (D11S4896E) at chromosomal region 11p15.5. Genomics 37: 253-256, 1996.

6. Johnstone LS, Graham SJ and Dziadek MA: STIM proteins: Integrators of signalling pathways in development, differentiation and disease. J Cell Mol Med 14: 1890-1903, 2010.

7. Suyama E, Wadhwa R, Kaur K, Miyagishi M, Kaul SC, Kawasaki $\mathrm{H}$ and Taira $\mathrm{K}$ : Identification of metastasis-related genes in a mouse model using a library of randomized ribozymes. J Biol Chem 279: 38083-38086, 2004.

8. Yang S, Zhang JJ and Huang XY: Orai1 and STIM1 are critical for breast tumor cell migration and metastasis. Cancer Cell 15: 124-134, 2009. 
9. Chen YF, Chiu WT, Chen YT, Lin PY, Huang HJ, Chou CY, Chang HC, Tang MJ and Shen MR: Calcium store sensor stromal-interaction molecule 1-dependent signaling plays an important role in cervical cancer growth, migration, and angiogenesis. Proc Natl Acad Sci USA 108: 15225-15230, 2011.

10. Yang N, Tang Y, Wang F, Zhang H, Xu D, Shen Y, Sun S and Yang G: Blockade of store-operated $\mathrm{Ca}(2+)$ entry inhibits hepatocarcinoma cell migration and invasion by regulating focal adhesion turnover. Cancer Lett 330: 163-169, 2013.

11. Umemura M, Baljinnyam E, Feske S, De Lorenzo MS, Xie LH, Feng X, Oda K, Makino A, Fujita T, Yokoyama U, et al: Store-operated $\mathrm{Ca} 2+$ entry (SOCE) regulates melanoma proliferation and cell migration. PLoS One 9: e89292,2014.

12. Sun J, Lu F, He H, Shen J, Messina J, Mathew R, Wang D, Sarnaik AA, Chang WC, Kim M, et al: STIM1- and Orail-mediated $\mathrm{Ca}(2+)$ oscillation orchestrates invadopodium formation and melanoma invasion. J Cell Biol 207: 535-548, 2014

13. Zhang Z, Liu X, Feng B, Liu N, Wu Q, Han Y, Nie Y, Wu K, Shi Y and Fan D: STIM1, a direct target of microRNA-185, promotes tumor metastasis and is associated with poor prognosis in colorectal cancer. Oncogene 34: 4808-4820, 2015.

14. Li W, Zhang M, Xu L, Lin D, Cai S and Zou F: The apoptosis of non-small cell lung cancer induced by cisplatin through modulation of STIM1. Exp Toxicol Pathol 65: 1073-1081, 2013.

15. Wang Y, Pan T, Wang H, Li L, Li J, Zhang C and Yang H: Silencing of TGIF attenuates the tumorigenicity of A549 cells in vitro and in vivo. Tumour Biol 37: 12725-12730, 2016.

16. Travis W, Brambilla E, Muller-Hermelink $\mathrm{H}$ and Harris C: World Health Organization classification of tumours. Pathology and Genetics Tumours of the Lung, Pleura, Thymus and Heart. Lyon, IARC Press, 2004

17. Wang Y, Zhai W, Wang H, Xia X and Zhang C: Benzo(a)pyrene promotes A549 cell migration and invasion through up-regulating Twist. Arch Toxicol 89: 451-458, 2015.

18. Bayne K: Revised guide for the care and use of laboratory animals available. American physiological society. Physiologist 39: 199, 208-211, 1996

19. Bratcher NA and Reinhard GR: Creative implementation of 3Rs principles within industry programs: Beyond regulations and guidelines. J Am Assoc Lab Anim Sci. 54: 133-138, 2015.

20. Hui L, Zhang S, Dong X, Tian D, Cui Z and Qiu X: Prognostic significance of twist and N-cadherin expression in NSCLC. PLoS One 8: e62171, 2013.

21. Bae GY, Choi SJ, Lee JS, Jo J, Lee J, Kim J and Cha HJ: Loss of E-cadherin activates EGFR-MEK/ERK signaling, which promotes invasion via the ZEB1/MMP2 axis in non-small cell lung cancer. Oncotarget 4: 2512-2522, 2013.

22. Gong L, Wu D, Zou J, Chen J, Chen L, Chen Y, Ni C and Yuan H: Prognostic impact of serum and tissue MMP-9 in non-small cell lung cancer: A systematic review and meta-analysis. Oncotarget 7: 18458-18468, 2016.

23. Yang X, Han M, Han H, Wang B, Li S, Zhang Z and Zhao W: Silencing snail suppresses tumor cell proliferation and invasion by reversing epithelial-to-mesenchymal transition and arresting $\mathrm{G} 2 / \mathrm{M}$ phase in non-small cell lung cancer. Int $\mathrm{J}$ Oncol 50: 1251-1260, 2017

24. Ye Z, Zhang X, Luo Y, Li S, Huang L, Li Z, Li P and Chen G: Prognostic values of Vimentin expression and its clinicopathological significance in non-small cell lung cancer: A meta-analysis of observational studies with 4118 cases. PLoS One 11: e0163162, 2016.

25. Zhong Z, Xia Y, Wang P, Liu B and Chen Y: Low expression of microRNA-30c promotes invasion by inducing epithelial mesenchymal transition in non-small cell lung cancer. Mol Med Rep 10: 2575-2579, 2014

26. Nitsche U, Stangel D, Pan Z, Schlitter AM, Esposito I, Regel I, Raulefs S, Friess H, Kleeff J and Erkan M: Periostin and tumor-stroma interactions in non-small cell lung cancer. Oncol Lett 12: 3804-3810, 2016

27. Shuman Moss LA and Stetler-Stevenson WG: Influence of stromal components on lung cancer carcinogenesis. J Carcinog Mutagen 13, 2013: doi: 10.4172/2157-2518.S13-008.

28. Zhang T, Xu J, Shen H, Dong W, Ni Y and Du J: Tumor-stroma ratio is an independent predictor for survival in NSCLC. Int J Clin Exp Pathol 8: 11348-11355, 2015.

29. Donnem T, Al-Saad S, Al-Shibli K, Delghandi MP, Persson M, Nilsen MN, Busund LT and Bremnes RM: Inverse prognostic impact of angiogenic marker expression in tumor cells versus stromal cells in non small cell lung cancer. Clin Cancer Res 13: 6649-6657, 2007.
30. Liotta LA and Kohn EC: The microenvironment of the tumour-host interface. Nature 411: 375-379, 2001

31. Sulzer MA, Leers MP, van Noord JA, Bollen EC and Theunissen PH: Reduced E-cadherin expression is associated with increased lymph node metastasis and unfavorable prognosis in non-small cell lung cancer. Am J Respir Crit Care Med 157: 1319-1323, 1998

32. Shibanuma H, Hirano T, Tsuji K, Wu Q, Shrestha B, Konaka C, Ebihara Y and Kato H: Influence of E-cadherin dysfunction upon local invasion and metastasis in non-small cell lung cancer. Lung Cancer 22: 85-95, 1998

33. Liu D, Huang C, Kameyama K, Hayashi E, Yamauchi A, Kobayashi S and Yokomise H: E-cadherin expression associated with differentiation and prognosis in patients with non-small cell lung cancer. Ann Thorac Surg 71: 949-955, 2001.

34. Moersig W, Horn S, Hilker M, Mayer E and Oelert H: Transfection of E-cadherin cDNA in human lung tumor cells reduces invasive potential of tumors. Thorac Cardiovasc Surg 50: 45-48, 2002

35. Asnaghi L, Vass WC, Quadri R, Day PM, Qian X, Braverman R, Papageorge AG and Lowy DR: E-cadherin negatively regulates neoplastic growth in non-small cell lung cancer: Role of Rho GTPases. Oncogene 29: 2760-2771, 2010.

36. Shi Y, Wu H, Zhang M, Ding L, Meng F and Fan X: Expression of the epithelial-mesenchymal transition-related proteins and their clinical significance in lung adenocarcinoma. Diagn Pathol 8: 89, 2013.

37. Dauphin M, Barbe C, Lemaire S, Nawrocki-Raby B, Lagonotte E, Delepine G, Birembaut P, Gilles C and Polette M: Vimentin expression predicts the occurrence of metastases in non small cell lung carcinomas. Lung Cancer 81: 117-122, 2013.

38. Tadokoro A, Kanaji N, Liu D, Yokomise H, Haba R, Ishii T, Takagi T, Watanabe N, Kita N, Kadowaki N and Bandoh S: Vimentin regulates invasiveness and is a poor prognostic marker in non-small cell lung cancer. Anticancer Res 36: 1545-1551, 2016.

39. Havel LS, Kline ER, Salgueiro AM and Marcus AI: Vimentin regulates lung cancer cell adhesion through a VAV2-Rac1 pathway to control focal adhesion kinase activity. Oncogene 34: 1979-1990, 2015

40. Rojiani MV, Alidina J, Esposito N and Rojiani AM: Expression of MMP-2 correlates with increased angiogenesis in CNS metastasis of lung carcinoma. Int J Clin Exp Pathol 3: 775-781, 2010.

41. Qian Z, Zhao X, Jiang M, Jia W, Zhang C, Wang Y, Li B and Yue W: Downregulation of cyclophilin A by siRNA diminishes non-small cell lung cancer cell growth and metastasis via the regulation of matrix metallopeptidase 9. BMC Cancer: 12: 442, 2012

42. He H, Zheng L, Sun YP, Zhang GW and Yue ZG: Steroidal saponins from Paris polyphylla suppress adhesion, migration and invasion of human lung cancer A549 cells via down-regulating MMP-2 and MMP-9. Asian Pac J Cancer Prev 15: 10911-10916, 2014.

43. Qian Q, Wang Q, Zhan P, Peng L, Wei SZ, Shi Y and Song Y: The role of matrix metalloproteinase 2 on the survival of patients with non-small cell lung cancer: A systematic review with meta-analysis. Cancer Invest 28: 661-669, 2010

44. Li J, Wang H, Ke H and Ni S: MiR-129 regulates MMP9 to control metastasis of non-small cell lung cancer. Tumour Biol 36: 5785-5790, 2015

45. Peinado H, Olmeda D and Cano A: Snail, Zeb and bHLH factors in tumour progression: An alliance against the epithelial phenotype? Nat Rev Cancer 7: 415-428, 2007.

46. Yanagawa J, Walser TC, Zhu LX, Hong L, Fishbein MC, Mah V, Chia D, Goodglick L, Elashoff DA, Luo J, et al: Snail promotes CXCR2 ligand-dependent tumor progression in non-small cell lung carcinoma. Clin Cancer Res 15: 6820-6829, 2009.

47. Liu J, Hu G, Chen D, Gong AY, Soori GS, Dobleman TJ and Chen XM: Suppression of SCARA5 by Snail1 is essential for EMT-associated cell migration of A549 cells. Oncogenesis 2: e73, 2013

48. Liu CW, Li CH, Peng YJ, Cheng YW, Chen HW, Liao PL, Kang JJ and Yeng MH: Snail regulates Nanog status during the epithelial-mesenchymal transition via the Smad1/Akt/GSK3 $\beta$ signaling pathway in non-small-cell lung cancer. Oncotarget 5: 3880-3894, 2014.

49. Merikallio H, Turpeenniemi-Hujanen T, Pääkkö P, Mäkitaro R, Riitta K, Salo S, Salo T, Harju T and Soini Y: Snail promotes an invasive phenotype in lung carcinoma. Respir Res 13: 104, 2012. 